COMMUNICATIONS IN

ANALYSIS AND GEOMETRY

Volume 13, Number 3, 511-525, 2005

\title{
Locally Holomorphic Maps Yield Symplectic Structures ${ }^{1}$
}

\author{
Robert E. GompF
}

\begin{abstract}
For a smooth map $f: X^{4} \rightarrow \Sigma^{2}$ that is locally modeled by holomorphic maps, the domain is shown to admit a symplectic structure that is symplectic on some regular fiber, if and only if $f^{*}[\Sigma] \neq 0$. If so, the space of symplectic forms on $X$ that are symplectic on all fibers is non-empty and contractible. The cohomology classes of these forms vary with the maximum possible freedom on the reducible fibers, subject to the obvious constraints. The above results are derived via an analogous theorem for locally holomorphic maps $f: X^{2 n} \rightarrow Y^{2 n-2}$ with $Y$ symplectic.
\end{abstract}

\section{Introduction.}

Research in the past dozen years has uncovered an intimate relationship between the differential topology of closed 4-manifolds and their symplectic structures. The latter are closed, non-degenerate 2 -forms, and have been shown to exist on many 4-manifolds (e.g., [3]). Many other 4-manifolds do not admit such structures [11], [10], [2], even though they may be homeomorphic (but not diffeomorphic) to symplectic manifolds. There has been much recent work aimed at understanding which 4-manifolds admit symplectic structures, as well as the range of values of the Chern class $c_{1}(\omega)$ and cohomology class $[\omega]$ of symplectic forms $\omega$ on a fixed manifold (e.g., [7], [9], [6]). For example, a closed 4-manifold admits a symplectic structure if and only if it admits a fibration-like structure called a Lefschetz pencil [1], [4]. In fact, a Lefschetz pencil (with fibers suitably intersecting the base locus) determines a symplectic structure up to isotopy, and a dense subset of all symplectic forms is realized this way up to scale. In this article, we use tools from [4] to investigate a related problem: We show that any smooth $f: X^{4} \rightarrow \Sigma^{2}$ that is locally modeled by holomorphic maps allows us to construct symplectic forms on $X$, provided that $f^{*}[\Sigma] \neq 0$. We show that the space of symplectic

\footnotetext{
${ }^{1}$ Partially supported by NSF grants DMS-9802533 and DMS-0102922.
} 
forms suitably compatible with $f$ is contractible, but that there is much freedom in the class $[\omega]$ when $f$ is sufficiently singular. We also investigate the corresponding problem in higher dimensions (Theorem 2.7).

The maps of interest are defined as follows.

Definition 1.1. A map $f: X^{2 n} \rightarrow Y^{2 m}$ between smooth, oriented manifolds is locally holomorphic if for each $x \in X$, there are smooth, orientationpreserving coordinate charts about $x$ and $f(x)$ (into $\mathbb{C}^{n}$ and $\mathbb{C}^{m}$, respectively) in which $f$ is given by a holomorphic map.

The fibers $f^{-1}(y)$ of a locally holomorphic map $f$ are homeomorphic to CWcomplexes. If each component of a compact fiber has (real) dimension 2, then the fiber is the image of some closed surface $F$ under a map that is a smooth embedding outside a finite subset of $F$ whose image we will denote by $K_{y} \subset X$. Near $K_{y}, f^{-1}(y)$ is cone-like. As in algebraic geometry, we will call the image of each connected component of $F$ an irreducible component of $f^{-1}(y)$. Note that some irreducible components may have multiplicities $>1$, so that they are contained in the critical set of $f$. In our case of primary interest ( $X$ compact, $n=2, m=1$ ), a (non-constant) locally holomorphic map is locally modeled by $f(z, w)=z^{d}$ where $d \geq 1$ is the (local) multiplicity of the fiber $f^{-1}(0)$, except on the finite set $K=\bigcup K_{y}$. Thus, the tangent spaces to the fibers form a real 2-plane bundle $L$ over $X-$ $K$, and this is canonically oriented (by the preimage orientation at regular points). There are simply connected 4 -manifolds that admit no complex structure, but do admit locally holomorphic maps to $S^{2}$ (e.g., [5]), even if we require all critical points to be of the simplest type (quadratic). One can construct more complicated examples, starting from any finite collection of (connected) singular fibers of holomorphic maps of a fixed generic fiber genus, by gluing together their tubular neighborhoods, and extending the resulting singular fibration from $D^{2}$ to $S^{2}$ by adding quadratic critical points to cancel the geometric monodromy. Note that each original singular fiber could have many irreducible components, with differing multiplicities.

We can now begin to state our main results.

Theorem 1.2. Let $X$ be a closed, oriented, connected 4-manifold with a locally holomorphic map $f: X \rightarrow \Sigma$ to a closed, oriented surface. Then, $X$ admits a symplectic structure that is symplectic on some regular fiber, if and only if $f^{*}[\Sigma] \neq 0 \in H_{\mathrm{dR}}^{2}(X)$.

The hypothesis $f^{*}[\Sigma] \neq 0$ is equivalent to requiring a generic fiber to be nontrivial in $H_{2}(X ; \mathbb{R})$. This is clearly necessary for the existence of symplectic 
structures on $X$ as above, since $\omega$ being symplectic on a generic fiber $f^{-1}(y)$ implies $\left\langle[\omega],\left[f^{-1}(y)\right]\right\rangle \neq 0$. (Sufficiency follows from Theorem 1.4 below.) However, the hypothesis $f^{*}[\Sigma] \neq 0$ is automatically satisfied whenever the generic fiber is not a torus (or disjoint union of tori). To see this, note that the above bundle $L$ over $X-K$ defines an Euler class $e(L) \in H^{2}(X-$ $K) \cong H^{2}(X)$. For a generic fiber, $\left\langle e(L), f^{-1}(y)\right\rangle=\left\langle e\left(T f^{-1}(y)\right), f^{-1}(y)\right\rangle=$ $\chi\left(f^{-1}(y)\right) \neq 0$ unless $f^{-1}(y)$ is a union of tori (since its components must be diffeomorphic). If we also require each fiber to have a neighborhood on which $f$ is globally modeled by a holomorphic map, then the classification of elliptic fibrations implies that $f^{*}[\Sigma] \neq 0$ unless $f$ is (up to blowups) a Seifert fibration (i.e., made from an honest torus bundle by adding smooth multiple fibers, and possibly composing with a branched covering map of $\Sigma$ to allow disconnected fibers).

We would like to study the space of symplectic structures on $X$ that are symplectic on all fibers of $f$. However, this condition makes no sense on the finite set $K$ where the fibers are singular. Instead, we use the following proposition, which is proved at the end of this section.

Proposition 1.3. A locally holomorphic map $f: X^{4} \rightarrow \Sigma^{2}$ canonically determines a complex bundle structure $J^{*}$ on $T X \mid K$. This is obtained by restricting the complex structures inherited from any choices of charts as in Definition 1.1, or more generally, by restricting any $C^{0}$ almost-complex structure $J$, defined on a neighborhood $U$ of $K$, for which the fibers of $f \mid U-K$ are $J$-holomorphic.

Recall that an almost-complex structure $J$ on $U$ is defined to be a complex structure on the tangent bundle $T U$, i.e., a bundle map covering $\operatorname{id}_{U}$ with $J \circ$ $J=-\mathrm{id}_{T U}$. We always assume almost-complex and symplectic structures respect the given orientations. Thus, in the proposition, $J$ determines the given orientation on $U$, and the tangent spaces to the regular fibers are $J$ complex lines in the preimage orientation. We only need almost-complex structures for a crude level of directional control in the tangent spaces. Thus, it is convenient to ignore differentiability and only require continuity of $J$, although we indicate where smoothness can be imposed for stronger conclusions.

Theorem 1.4. For $f: X \rightarrow \Sigma$ as in Theorem 1.2 with $f^{*}[\Sigma] \neq 0$, let $\mathcal{S}$ be the space of symplectic forms on $X$ that are symplectic on the (canonically oriented) fibers of $f \mid X-K$ and tame $J^{*}$ on $K$. Then:

a) $\mathcal{S}$ is non-empty and contractible. 
b) $\mathcal{S}$ is also characterized as being the space of symplectic forms taming global $C^{0}$ (or $C^{\infty}$ ) almost-complex structures $J$ on $X$ such that each regular fiber of $f$ (and hence, each fiber of $f \mid X-K$ ) is $J$-holomorphic.

For taming, see Definition 2.1. There is considerable freedom to choose the topology on $\mathcal{S}$. For example, we can choose any $C^{k}$-topology or Sobolev topology in between. See [4, Theorem 2.11(b) and subsequent discussion] for further details. Note that Theorem 1.4 immediately implies Theorem 1.2.

To understand our freedom to choose the cohomology class $[\omega] \in$ $H_{\mathrm{dR}}^{2}(X)$, first note some obvious constraints: If $F_{1}, \ldots, F_{n}$ are the (canonically oriented) irreducible components of a single connected component of a fiber, and $F_{i}$ has multiplicity $m_{i}$, then (as we will see) $\sum m_{i}\left[F_{i}\right] \in H_{2}(X ; \mathbb{R}$ ) must be a rational multiple of the generic fiber class $\left[f^{-1}(y)\right]$ (where the multiplier $q$ is 1 if the fibers are connected). Thus, we must have $\sum m_{i}\left\langle\omega, F_{i}\right\rangle=q\left\langle\omega, f^{-1}(y)\right\rangle$. Furthermore, for $\omega \in \mathcal{S}$, each $F_{i}-K$ must be symplectic, so $\left\langle\omega, F_{i}\right\rangle>0$. These turn out to be the only constraints on the areas $\left\langle\omega, F_{i}\right\rangle$.

Theorem 1.5. For fixed $J$ as in Theorem 1.4(b), there is a form $\omega \in \mathcal{S}$ taming $J$, such that the areas $\left\langle\omega, F_{i}\right\rangle$ of irreducible components of fibers of $f$ take any preassigned values subject to the above constraints.

That is, the (suitably weighted) areas of the irreducible components of each connected component of each fiber can be distributed in any preassigned manner.

Since $\mathcal{S}$ is connected, all $\omega \in \mathcal{S}$ have the same Chern class $c_{1}(\omega)$. We can compute this class using some $J$ as in Theorem 1.4(b) and the $J$-complex line bundle $L$ over $X-K$ of tangent planes to fibers. If the critical set of $f$ in $X$ is finite, then by definition $c_{1}(\omega)=c_{1}(T X, J)=c_{1}\left(L \oplus f^{*} T \Sigma\right)=$ $e(L)+\chi(\Sigma) f^{*}[\Sigma]=e(L)+\chi(\Sigma) P D\left[f^{-1}(y)\right]$ for a generic fiber $f^{-1}(y)$ (where $\mathrm{PD}$ is Poincaré duality). The general case is obtained by adding a term $\left(1-m_{i}\right) P D\left[F_{i}\right]$ for each irreducible component $F_{i}$ of multiplicity $m_{i}$ in each singular fiber. (A vector field on $\Sigma$ which is non-zero near the critical values of $f$ lifts to one on $L^{\perp}$ with index $1-m_{i}$ near $F_{i}$, since sources (index 1 ) at critical values lift to sources.)

We prove Theorems 1.4 and 1.5 in Section 3, using tools from [4]. Since these tools are applicable in arbitrary dimensions, we proceed by first proving a theorem that holds in all dimensions. This theorem says that under suitable hypotheses, a locally holomorphic map to a symplectic manifold, with 2-dimensional fibers, determines a deformation class of symplectic structures on its domain (a deformation being a smooth family $\omega_{t}, 0 \leq t \leq 1$, 
of symplectic forms). The precise statement (Theorem 2.7) requires further definitions, and is the subject of the next section.

Proof of Proposition 1.3. For a locally holomorphic map $f: X^{4} \rightarrow \Sigma^{2}$ and $x \in K$ a singular point of a fiber, we must show that all almost-complex structures defined near $K$, making the fibers of $f J$-holomorphic, have the same restriction $J^{*}$ to $T_{x} X$. Identify a 4-ball neighborhood $U$ of $x$ in $X$ with a neighborhood of 0 in $\mathbb{C}^{2}$ as in Definition 1.1. It is not hard to show that a linear complex structure on $\mathbb{R}^{2 n}(n \neq 1)$ is determined by its 1-dimensional (oriented) complex subspaces [4, Lemma 4.4(a)], so it suffices to show that every complex line at 0 in $\mathbb{C}^{2}$ can be written as lim ker $d f_{x_{i}}$ for some sequence of regular points $x_{i} \rightarrow 0$. We can assume $U \cap K=\{x\}$, so the bundle $L$ of tangents to fibers of $f$ is defined on $U-\{0\} \subset \mathbb{C}^{2}$ and determines a holomorphic map $\varphi: U-\{0\} \rightarrow \mathbb{C P}^{1}$, whose homogeneous coordinates $\varphi_{i}$, $i=1,2$, are obtained from $\frac{\partial f}{\partial z_{i}}$ by removing common factors (to remove the singularities along the critical set). Up to homotopy, continuous maps $U-\{0\} \simeq S^{3} \rightarrow \mathbb{C P}^{1}$ are classified by $\pi_{3}\left(S^{2}\right) \cong \mathbb{Z}$, and we can compute this integer invariant $h(\varphi)$ by the Thom-Pontrjagin construction: The fibers $\varphi_{i}^{-1}(0)$ of $\varphi$ each intersect a small $S^{3} \subset U-\{0\}$ in an oriented link $L_{i}$ (possibly with multiplicities), and $h(\varphi)=\ell k\left(L_{1}, L_{2}\right)$ is their linking number. If $0 \notin \varphi_{i}^{-1}(0)$ for some $i$, then $\varphi$ determines a holomorphic map $U-\{0\} \rightarrow \mathbb{C}$ (for $U$ sufficiently small), so $L$ extends holomorphically over 0 by Hartogs' Theorem. But $L \mid U-\{0\}$ is tangential to the fibers of $f$, so $L$ is integrable on $U$ (since the Frobenius condition is closed), and $f^{-1}(0)$ is a smooth leaf of the foliation, contradicting our assumption that $x \in K$. Thus, $0 \in$ $\varphi_{1}^{-1}(0) \cap \varphi_{2}^{-1}(0)$, so $h(\varphi)=\ell k\left(L_{1}, L_{2}\right)=\varphi_{1}^{-1}(0) \cdot \varphi_{2}^{-1}(0)>0$. In particular, $\varphi$ is surjective on arbitrarily small 3 -spheres about 0 , and so the required sequence $\left(x_{i}\right)$ is easy to construct.

\section{Arbitrary dimensions.}

We begin with some terminology for relating symplectic and complex structures.

Definition 2.1. [4] Let $T: V \rightarrow W$ be a linear transformation between finite-dimensional real vector spaces, and let $\omega$ be a skew-symmetric bilinear form on $W$. A linear complex structure $J: V \rightarrow V$ will be called $(\omega, T)$-tame if $T^{*} \omega(v, J v)>0$ for all $v \in V-\operatorname{ker} T$. If, in addition, $T^{*} \omega$ is $J$-invariant (i.e., $T^{*} \omega(J v, J w)=T^{*} \omega(v, w)$ for all $\left.v, w \in V\right)$, we will call $J(\omega, T)$ compatible. For a $C^{1}$-map $f: X \rightarrow Y$ between manifolds, with a 2-form $\omega$ 
on $Y$, an almost-complex structure $J$ on $X$ will be called $(\omega, f)$-tame (resp. $(\omega, f)$-compatible $)$ if it is $\left(\omega, d f_{x}\right)$-tame (resp. $\left(\omega, d f_{x}\right)$-compatible) for each $x \in X$. If $T=\mathrm{id}_{V}$ or $f=\mathrm{id}_{X}$, we will shorten the terminology to $\omega$-tame and $\omega$-compatible.

The last sentence of the definition is standard terminology. If $\omega$ tames some $J$ (i.e., $T=\operatorname{id}_{V}$ and $J$ is $\omega$-tame), then $\omega$ is positive on all $J$-complex lines, and it is obviously non-degenerate (that is, every $v \neq 0$ pairs non-trivially with something), so a closed, taming 2 -form $\omega$ is automatically symplectic. Both the taming and compatibility conditions are preserved under taking convex combinations $\sum t_{i} \omega_{i}\left(\right.$ all $\left.t_{i} \geq 0, \sum t_{i}=1\right)$ for fixed $J$. However, taming is more natural for our purposes than compatibility, since $\omega$-taming is preserved under small perturbations of $\omega$ and $J$. It is an open question whether compatibility can be replaced by taming throughout the paper. (It can be done, for example, if Question 4.3 of [4] has an affirmative answer.) In the special case where $\operatorname{dim}_{\mathbb{R}} W=2$ and $\omega$ respects a preassigned orientation on $W$, then $\omega$ is determined up to positive scale, so $(\omega, T)$-taming and $(\omega, T)$ compatibility are equivalent, independent of choice of $\omega$, and equivalent to the condition that $\operatorname{ker} T$ (in the preimage orientation if $T \neq 0$, where $J$ orients $V$ ) be a $J$-complex subspace of $V$. In particular:

Proposition 2.2. For $f: X \rightarrow \Sigma$ as in Theorem 1.4, $J$ satisfies the condition of (b) (every regular fiber is $J$-holomorphic) if and only if $J$ is $\left(\omega_{\Sigma}, f\right)$ tame (or $\left(\omega_{\Sigma}, f\right)$-compatible), where $\omega_{\Sigma}$ is any (positive) area form on $\Sigma$.

Such structures $J$ are easy to construct: Split $T(X-K)$ as $L \oplus L^{\prime}$ where $L$ is tangent to the fibers and $L^{\prime}$ is complex near $K$ for some locally defined complex structure as in Definition 1.1, then declare $L$ and $L^{\prime}$ to be complex line bundles. However, it is more difficult to arrange $J$ to tame a preassigned $\omega \in \mathcal{S}$. Thus, while it is easy to see that any $\omega$ as in Theorem 1.4(b) lies in $\mathcal{S}$, the converse takes more work.

In higher dimensions, we need to strengthen our local holomorphicity condition:

Definition 2.3. For a symplectic form $\omega$ on $Y$, a locally holomorphic map $f: X \rightarrow Y$ is called $\omega$-compatibly locally holomorphic if the charts in Definition 1.1 can be chosen so that $\omega$ is compatible with the standard complex structure on $\mathbb{C}^{m}$.

This is automatically satisfied when $\operatorname{dim}_{\mathbb{R}} Y=2$, or for holomorphic charts on a Kähler $(Y, \omega)$. It implies that the corresponding local complex structures on $X$ are $(\omega, f)$-compatible. 
We also need a technical condition from [4] to control the behavior of fibers near critical points; this is again vacuous for non-constant locally holomorphic maps $f: X^{4} \rightarrow \Sigma^{2}$. Suppose $E, F \rightarrow X$ are real vector bundles over a metrizable topological space, and $T: E \rightarrow F$ is a section of the bundle $\operatorname{Hom}(E, F)$. In our main application, these will be induced by a $C^{1}$-map $f: X \rightarrow Y$ between manifolds, with $T=d f: T X \rightarrow f^{*} T Y$. Motivated by this example, we call a point $x \in X$ regular if $T_{x}: E_{x} \rightarrow F_{x}$ is onto and critical otherwise. Let $P \subset E$ be the closure $\operatorname{cl}\left(\bigcup \operatorname{ker} T_{x}\right)$, where $x$ varies over all the regular points of $T$ in $X$, and let $P_{x}=P \cap E_{x}$. Thus, $P_{x}=\operatorname{ker} T_{x}$ if $x$ is regular, and otherwise $P_{x} \subset \operatorname{ker} T_{x}$ consists of limits of sequences of vectors annihilated by $T$ at regular points.

Definition 2.4. [4, Definition 2.2] A point $x \in X$ is wrapped if span $P_{x}$ has (real) codimension at most 2 in $\operatorname{ker} T_{x}$.

In our application, this condition automatically holds away from fiber components with multiplicities:

Proposition 2.5. [4, Proposition 2.3] Suppose that in a neighborhood of a critical point $x \in X, T$ is given by $d f$, for some holomorphic map $f: U \rightarrow$ $\mathbb{C}^{n-1}$ with $U$ open in $\mathbb{C}^{n}$. If each fiber $f^{-1}(y)$ intersects the critical set of $f$ in at most a finite set, then $x$ is wrapped. In fact, $P_{x}=\operatorname{ker} T_{x}$.

Note that the proposition becomes false without the finiteness hypothesis, e.g., $n=3, f(x, y, z)=\left(x^{2}, y^{2}\right)$ at $(0,0,0)$. For $n=2, P_{x}=\operatorname{ker} T_{x}$ unless $f$ is constant or $x$ is a smooth point of a fiber component with multiplicity $>1$, cf. proof of Proposition 1.3, but every point of a non-constant locally holomorphic map $f: X^{4} \rightarrow \Sigma^{2}$ will be wrapped (since regular points are dense, implying $\operatorname{dim}_{\mathbb{R}} \operatorname{span} P_{x} \geq 2$ everywhere).

To state the main theorem, we must first orient the fibers.

Lemma 2.6. Let $f: X^{2 n} \rightarrow Y^{2 n-2}$ be a locally holomorphic map, all of whose fibers have real dimension 2. Fix $y \in Y$.

a) For each $x \in f^{-1}(y)-K_{y}$, there is a sequence $x_{i} \rightarrow x$ of regular points in $X$ for which $T_{x} f^{-1}(y)=\lim \operatorname{ker} d f_{x_{i}}$.

b) The surface $f^{-1}(y)-K_{y}$ is canonically oriented, by sequences as in (a) and the preimage orientation on each ker $d f_{x_{i}}$. 
Proof. For (a), it suffices to find such a sequence $\left(x_{i}\right)$ for each $x$ in a dense subset of $f^{-1}(y)$. After restricting to suitable neighborhoods, we may assume $f$ is holomorphic. The critical values of $f$ lie in a local subvariety of $Y$ with positive codimension, so there is a holomorphic disk $D$ in $Y$ (with $\operatorname{dim}_{\mathbb{C}} D=1$ ) centered at $y$ and disjoint from the critical set elsewhere. After suitably shrinking $D$, we conclude that $D \subset f(X)$. (Otherwise $f(X)$ lies in a variety of positive codimension, so $f$ has fibers of real dimension $>2$.) Thus, $f^{-1}(D)$ is locally a variety of complex dimension 2 . After we resolve the singularities of $f^{-1}(D)$, the required sequences obviously exist on the resulting complex surface, since generically $f$ is given locally by $f(z, w)=z^{d}$ with fibers parallel to the $w$-axis. The sequences push down to $f^{-1}(D)$ as required. (This follows a suggestion of S. Keel.)

To prove (b), note that the preimage orientation on each ker $d f_{x_{i}}$ is also its complex orientation (for the complex structure determined by our choice of charts in Definition 1.1). These then limit to the complex orientation on $T_{x} f^{-1}(y)$, which is independent of the choice of $\left(x_{i}\right)$ converging to $x$. Since this orientation is now determined by a fixed $x_{i} \rightarrow x$, it is also independent of our choice of charts, and hence global and canonical.

Theorem 2.7. Let $X^{2 n}, Y^{2 n-2}$ be closed, oriented manifolds with a symplectic form $\omega_{Y}$ on $Y$ and an $\omega_{Y}$-compatibly locally holomorphic map $f: X \rightarrow Y$, all of whose fibers have real dimension 2. Suppose there is a class $c \in H_{\mathrm{dR}}^{2}(X)$ evaluating positively on each irreducible component of each fiber of $f$ (canonically oriented). If $n \geq 3$, assume that all critical points of $f$ are wrapped. Then:

a) $X$ admits a symplectic structure. In fact, there is a unique deformation class of symplectic forms on $X$ containing representatives that tame $\left(\omega_{Y}, f\right)$-compatible, global $C^{0}$ almost-complex structures $J$. This still holds if we require $J$ to be $C^{\infty}$, with a $C^{\infty}, \omega_{Y}$-compatible structure on $f^{*} T Y$ making $d f J$-complex.

b) For any fixed $C^{0},\left(\omega_{Y}, f\right)$-tame $J$ on $X$, the convex open cone in $H_{\mathrm{dR}}^{2}(X)$ consisting of classes of symplectic forms taming $J$ contains all classes $t c+f^{*}\left[\omega_{Y}\right]$ for $c$ as above and $t>0$ sufficiently small (depending on $c$ ).

Remark 1. When $n=3$, the condition of wrapped critical points can be dropped, and existence and (b) still follow. In fact the entire theorem still holds then, if we also require the structures $J$ in (a) to be $\left(\omega_{Y}, d f\right)$-extendible as in [4], along preassigned sequences converging to the unwrapped critical 
points. The proof is identical to that of Theorem 2.7, once we augment Lemma 3.2 by [4, Addendum 3.3] and note that the charts of Definition 2.3 are automatically $\left(\omega_{Y}, d f\right)$-extendible. The resulting deformation class in (a) is independent of the choice of sequences, since any $J$ satisfying all the conditions of $(\mathrm{a})$ is $\left(\omega_{Y}, d f\right)$-extendible for all sequences.

\section{Proofs.}

It remains to prove Theorems 2.7, 1.4 and 1.5. We need two results from [4]. The first allows us to construct symplectic structures on the domain $X$ of a map to a symplectic manifold, in the presence of a suitable almost-complex structure $J$ on $X$. The second allows us to construct $J$.

Theorem 3.1. [4] Let $f: X \rightarrow Y$ be a smooth map between closed manifolds. Suppose that $\omega_{Y}$ is a symplectic form on $Y$, and $J$ is a continuous, $\left(\omega_{Y}, f\right)$-tame almost-complex structure on $X$. Fix a class $c \in H_{\mathrm{dR}}^{2}(X)$. Suppose that for each $y \in Y, f^{-1}(y)$ has a neighborhood $W_{y}$ in $X$ with a closed 2 -form $\eta_{y}$ such that $\left[\eta_{y}\right]=c \mid W_{y} \in H_{\mathrm{dR}}^{2}\left(W_{y}\right)$ and such that $\eta_{y}$ tames $J$ on each of the complex subspaces ker $d f_{x}, x \in W_{y}$. Then, there is a closed 2form $\eta$ on $X$ with $[\eta]=c \in H_{\mathrm{dR}}^{2}(X)$, and such that for all sufficiently small $t>0$ the form $\omega_{t}=t \eta+f^{*} \omega_{Y}$ on $X$ tames $J$ (and hence is symplectic).

This is [4, Theorem 3.1], restricted to the case with $C=\emptyset$. The main idea of the proof goes back to Thurston [12] in the case of surface bundles. We cannot directly splice the forms $\eta_{y}$ by a partition of unity, without losing closure of the forms. Instead, we subtract off a global representative $\zeta$ of $c$ to obtain exact forms, then splice via the corresponding 1-forms and add $\zeta$ back in. The resulting closed 2-form $\eta$ is non-degenerate on each ker $d f_{x}$ by convexity of the $J$-taming condition. (This use of $J$ to control non-degeneracy is the innovation allowing us to deal with general critical points of $f$.) The form $f^{*} \omega_{Y}$ provides non-degeneracy for $\omega_{t}$ in the remaining directions.

Splicing together local almost-complex structures is harder. Here, we only state a simplified version of [4, Lemma 3.2] without proof. As preceding Definition 2.4, we let $E, F \rightarrow X$ be real vector bundles over a metrizable space, with fiber dimensions $2 n$ and $2 n-2$ respectively, and this time equipped with fiber orientations. We again fix a section $T: E \rightarrow F$ of $\operatorname{Hom}(E, F)$. A 2-form on $E$ or $F$ means a continuous choice of skewsymmetric bilinear forms on the fibers.

Lemma 3.2. [4] For $E, F \rightarrow X$ and $T$ as above with $X$ compact, suppose that the regular points of $T$ are dense in $X$, and let $\omega_{F}$ be a non-degenerate 
2-form on $F$ (inducing the given fiber orientation). Suppose that each $x \in$ $X$ has a neighborhood $W_{x}$ with an $\left(\omega_{F}, T\right)$-compatible complex (bundle) structure on $E \mid W_{x}$.

a) If $n \geq 3$, assume each critical point of $T$ is wrapped. Then, the space $\mathcal{J}$ of $\left(\omega_{F}, T\right)$-compatible complex structures on $E$ is non-empty and contractible (in the $C^{0}$-topology). For any $\left(\omega_{F}, T\right)$-compatible structure $J_{C}$ defined near a closed subset $C \subset X$, there are elements of $\mathcal{J}$ agreeing with $J_{C}$ on $E \mid C$.

b) Fix a 2-form $\omega_{E}$ on $E$ and a complex structure on $F$. Then, (a) remains true if each complex structure on $E$ and its restrictions is required to be $\omega_{E}$-tame and to make $T$ complex linear.

Proof of Theorem 2.7. For $f: X \rightarrow Y$ as given, Lemma 3.2(a) (with $\left.T=d f: T X \rightarrow f^{*} T Y\right)$ implies that the space $\mathcal{J}$ of $\left(\omega_{Y}, f\right)$-compatible almost-complex structures on $X$ is non-empty and contractible. To see this, note that regular points of $f$ are dense (e.g., by Lemma 2.6(a)), and that the local complex structures on $X$ given by Definition 2.3 and subsequent text are automatically $\left(\omega_{Y}, f\right)$-compatible. The relevant part of the existence proof of Lemma 3.2(a) is smooth, and automatically produces elements of $\mathcal{J}$ satisfying all the conditions of Theorem 2.7(a). Now, we check that each (canonically oriented) fiber $f^{-1}(y)-K_{y}$ is a $J$-holomorphic curve for each $J \in \mathcal{J}$, or more generally, for any $\left(\omega_{Y}, f\right)$-tame (positively oriented) $C^{0}$ almost-complex structure $J$ on $X$. This follows from Lemma 2.6 once we verify that each ker $d f_{x}$, with $x$ regular, is a $J$-complex line in the preimage orientation. But Definition 2.1 implies $\operatorname{ker} d f_{x}$ is $J$-invariant, so $T_{f(x)} Y \cong$ $T_{x} X /$ ker $d f_{x}$ inherits an $\omega_{Y}$-tame complex structure $f_{*} J$. After homotoping $\omega_{Y}$ through taming structures to one that is compatible with $f_{*} J$, it is easy to verify that $f_{*} J$ induces the same orientation on $T_{f(x)} Y$ as $\omega_{Y}$. Thus, $J$ induces the preimage orientation on ker $d f_{x}$.

For any fixed $\left(\omega_{Y}, f\right)$-tame $J$ on $X$, such as any $J \in \mathcal{J}$, we wish to apply Theorem 3.1 with $c$ as given. The argument follows the method of $[4$, Theorem 2.11(b)], but with complications arising from fibers with irreducible components lying in the critical set of $f$. For $y \in Y$, let $K_{y}^{\prime} \supset K_{y}$ be a finite subset of $f^{-1}(y)$ intersecting each irreducible component non-trivially, and let $\sigma$ be a closed 2-form taming $J$ on some neighborhood $W$ of $K_{y}^{\prime}$ in $X$. Since $f^{-1}(y)-K_{y}$ is $J$-holomorphic, $\sigma \mid\left(f^{-1}(y)-K_{y}\right) \cap W$ is a positive area form, and we can extend this to an area form on $f^{-1}(y)-K_{y}$. We can easily arrange $\int_{F_{i}} \sigma=\left\langle c, F_{i}\right\rangle>0$ for each irreducible component $F_{i}$ of 
$f^{-1}(y)$. Let $F^{*} \subset f^{-1}(y)$ be a compact surface with boundary, obtained by deleting a neighborhood of $K_{y}^{\prime}$ whose closure lies in $W$. Since $F^{*}$ is $J$ holomorphic, $T F^{*} \subset T X \mid F^{*}$ has a complementary complex subbundle $\nu F^{*}$. This bundle is trivial since $F^{*}$ has no closed components, so we can use it to identify a tubular neighborhood $N$ of $F^{*}$ with $F^{*} \times \mathbb{C}^{n-1}$, by a map that is $J$-holomorphic on $T N \mid F^{*}$. After shrinking $W$, we may assume $N \cap W$ corresponds to $\left(F^{*} \cap W\right) \times \mathbb{C}^{n-1}$. The product form $\tau=\pi_{1}^{*}\left(\sigma \mid F^{*}\right)+\pi_{2}^{*} \omega_{\mathbb{C}^{n-1}}$ on $N$ tames $J$ on $T N \mid F^{*}$. The form $\sigma-\tau$ on $N \cap W$ is closed, and it vanishes on $F^{*} \cap W$, so it is exact. Choose a 1 -form $\alpha$ on $N \cap W$ with $d \alpha=\sigma-\tau$. Subtracting the closed form $\pi_{1}^{*}\left(\alpha \mid F^{*} \cap W\right)$ from $\alpha$ if necessary, we can arrange $\alpha \mid F^{*} \cap W=0$. Now, the 1-form $\alpha: T(N \cap W) \rightarrow \mathbb{R}$ restricts to a fiberwiselinear map $\nu F^{*} \mid F^{*} \cap W \rightarrow \mathbb{R}$; by our identification of this bundle with $N \cap W$, we obtain a smooth map $\varphi: N \cap W \rightarrow \mathbb{R}$ with $\varphi \mid F^{*} \cap W=0$ and $d \varphi=\alpha$ on $T X \mid F^{*} \cap W$. Subtracting $d \varphi$ from $\alpha$, we arrange $\alpha=0$ on $T X \mid F^{*} \cap W$. Choose a map $\rho: F^{*} \rightarrow[0,1]$ with $\rho=1$ near $\partial F^{*}$ and $\rho=0$ outside $W$, and let $\eta_{y}=\tau+d\left(\left(\rho \circ \pi_{1}\right) \alpha\right)$ on $N$. Then, the closed form $\eta_{y}$ agrees with $\tau$ outside $W$ and extends as $\sigma$ near $f^{-1}(y)-F^{*}$. Furthermore, $\eta_{y}=\sigma$ on $f^{-1}(y)-K_{y}$. On $T X \mid F^{*} \cap W, d\left(\rho \circ \pi_{1}\right) \wedge \alpha=0$ so $\eta_{y}=\tau+\rho d \alpha$ is a convex combination of the $J$-taming forms $\tau$ and $\sigma$. Thus, $\eta_{y}$ tames $J$ on $T X \mid f^{-1}(y)$ and hence, on a regular neighborhood $W_{y}$ of $f^{-1}(y)$ in $X$. Since $\int_{F_{i}} \eta_{y}=\int_{F_{i}} \sigma=\left\langle c, F_{i}\right\rangle$ for each $F_{i}$, so $\left[\eta_{y}\right]=c \mid W_{y}$, Theorem 3.1 gives us a global closed 2-form $\eta$ on $X$ with $[\eta]=c$ and $\omega=t \eta+f^{*} \omega_{Y}$ taming $J$ (hence, symplectic) for any sufficiently small $t>0$. As required, $[\omega]=t c+f^{*}\left[\omega_{Y}\right]$, so we have proved (b) and the existence part of (a).

To prove uniqueness in (a), we must find a deformation between any preassigned pair $\omega_{s}, s=0,1$, of forms taming structures $J_{s} \in \mathcal{J}$. Since $\mathcal{J}$ is contractible, we can extend to a family $J_{s} \in \mathcal{J}, 0 \leq s \leq 1$. For each $s \in(0,1)$, Part (b) (with any convenient choice of $c$ ) yields a symplectic form $\omega_{s}$ taming $J_{s}$ (not necessarily continuous in $s$ ). Since the taming condition is open and $X$ is compact, each $\omega_{s}, s \in[0,1]$, tames $J_{t}$ for $t$ in some neighborhood of $s$. Convexity of the taming and closure conditions now allows us to smooth the family $\omega_{s}$ by a partition of unity on $[0,1]$, to obtain the required deformation of symplectic forms.

Proof of Theorem 1.5. For $f: X \rightarrow \Sigma$ and $J$ as in Theorem 1.4, we wish to construct symplectic structures using Theorem 2.7(b). We have already observed (following Definition 2.3) that $f$ is $\omega_{\Sigma}$-compatibly locally holomorphic for any $\omega_{\Sigma}$, and (Proposition 2.2) $J$ is $\left(\omega_{\Sigma}, f\right)$-tame. Since $f^{*}[\Sigma] \neq 0, f$ is surjective (onto a component of $\Sigma$ ), so local holomorphicity 
implies each fiber is 2-dimensional. To construct a suitable class $c$, we invoke the following lemma, whose proof is given at the end of the paper.

Lemma 3.3. For an $n \times n$ real, symmetric matrix $A=\left[a_{i j}\right]$, let $G_{A}$ denote the graph with $n$ vertices $v_{1}, \ldots, v_{n}$, and an edge between any two distinct vertices $v_{i}, v_{j}$ whenever $a_{i j} \neq 0$. Suppose that (a) $G_{A}$ is connected, (b) $a_{i j} \geq 0$ whenever $i \neq j$, and (c) there are positive real numbers $m_{1}, \ldots, m_{n}$ such that $\sum_{i=1}^{n} m_{i} a_{i j} \leq 0$ for all $j$. Fix a choice of such numbers $m_{i}$. Then the hypothesis (d), that the inequality in (c) is strict for some $j$, implies $\operatorname{rank} A=n$. If (d) is not satisfied, then $\operatorname{rank} A=n-1$.

Let $F_{1}, \ldots, F_{n}$ denote the irreducible components comprising a single connected component of a singular fiber $f^{-1}\left(y_{0}\right)$. For a disk $D$ about $y_{0}$ containing no other critical values, and $y \neq y_{0}$ in $D$, let $\varphi \in H_{2}(X ; \mathbb{R})$ be the homology class of the union of all components of $f^{-1}(y)$ lying in the same component of $f^{-1}(D)$ as $\bigcup_{i=1}^{n} F_{i}$. Then, $\varphi=\sum_{i=1}^{n} m_{i}\left[F_{i}\right]$, where $m_{i}>0$ is the multiplicity of $F_{i}$. Furthermore, $\varphi$ is a positive rational multiple of $\left[f^{-1}(y)\right]=P D f^{*}[\Sigma]$. (Since $f$ restricts to a fiber bundle with connected total space away from the finite set of critical values in $\Sigma$, any two components of generic fibers are isotopic.) If $A=\left[a_{i j}\right]$ is the symmetric $n \times n$ matrix for which $a_{i j}$ is the intersection number $F_{i} \cdot F_{j}$, then $\sum_{i=1}^{n} m_{i} a_{i j}=\varphi \cdot F_{j}=0$ for all $j$ (since $f^{-1}(y) \cap F_{j}=\emptyset$ ). Thus, $A$ satisfies (a)-(c) but not (d) of the above lemma, so rank $A=n-1$. For any $s_{1}, \ldots, s_{n} \in \mathbb{R}$ with $\sum m_{i} s_{i}=0$, we can now find constants $r_{1}, \ldots, r_{n} \in \mathbb{R}$ such that $\psi=\sum r_{i}\left[F_{i}\right]$ satisfies $\psi \cdot F_{j}=s_{j}$ for all $j$. Clearly, $\psi$ pairs trivially with every other irreducible component of every fiber. Since $\left[f^{-1}(y)\right] \neq 0$ by hypothesis, we can find a class $c_{0}$ in $H_{\mathrm{dR}}^{2}(X)$ with $\left\langle c_{0}, f^{-1}(y)\right\rangle>0$ (so $c_{0}$ is positive on each component of each generic fiber). After adding the Poincaré dual of a suitable class $\psi$ for each connected component of each singular fiber, we obtain a class $c$ realizing any preassigned values on irreducible components of fibers, subject to the condition that $\sum_{i=1}^{n} m_{i}\left\langle c, F_{i}\right\rangle=\left\langle c_{0}, \varphi\right\rangle$ for each connected component of each singular fiber. Choosing these values to be positive, we apply Theorem 2.7(b) to obtain symplectic forms taming $J$ (hence in $\mathcal{S}$ as characterized in Theorem 1.4(b)). Since $\left\langle f^{*}\left[\omega_{\Sigma}\right], F_{i}\right\rangle=0$ for each $F_{i}$, we obtain the required flexibility of $[\omega]$.

Proof of Theorem 1.4. By Proposition 2.2 and subsequent text, a structure $J$ satisfies the condition of Theorem 1.4(b) if and only if it is $\left(\omega_{\Sigma}, f\right)$ compatible, such structures $J$ are easy to construct, and any $\omega$ as in (b) lies 
in $\mathcal{S}$. Thus, $\mathcal{S} \neq \emptyset$ by Theorem 1.5. It remains to show that $\mathcal{S}$ is contractible and any $\omega \in \mathcal{S}$ tames some $J$ as in (b). As in the contractibility proof of [4, Theorem 2.11(b)], a map of a sphere $S^{m} \rightarrow \mathcal{S}$ can be interpreted as a family $\omega$ of symplectic forms on the fibers of the trivial bundle $S^{m} \times X$. Let $L \rightarrow S^{m} \times(X-K)$ denote the bundle of tangent spaces to fibers of $f$, and let $L^{\perp}$ denote its orthogonal complement in $S^{m} \times T(X-K)$ with respect to the given family $\omega$. (Then, $L \oplus L^{\perp}=S^{m} \times T(X-K)$ since $\omega$ is symplectic on each fiber of $f$, and $\omega$ is non-degenerate on each subbundle.) Let $J_{0}$ be a complex structure near $K$ induced by Definition 1.1, pulled back to the $X$ fibers of $S^{m} \times X$. Since $J_{0} \mid S^{m} \times K=J^{*}$ is $\omega$-tame, $J_{0}$ is $\omega$-tame near some compact subset $\hat{X} \subset S^{m} \times X$ whose interior contains $S^{m} \times K$. Since the fibers of $f$ are $J_{0}$-holomorphic on $\hat{X}$, we can obtain a new complex structure $J_{1}$ on $\left(S^{m} \times T X\right) \mid\left(\hat{X}-S^{m} \times K\right)$ by identifying $L^{\perp}$ there with $S^{m} \times T X / L$ and summing the resulting complex structure on $L^{\perp}$ with the given one on $L$. Then, $J_{1}$ is $\omega$-tame by $\omega$-orthogonality of the line bundle $L^{\perp}$, and $f$ is both $J_{0^{-}}$and $J_{1}$-holomorphic for the same complex structure on $S^{m} \times f^{*} T \Sigma \mid \hat{X}$. Thus, we can interpolate between $J_{0}$ and $J_{1}$ on $\hat{X}$ using Lemma 3.2(b): Set $T=\operatorname{id}_{S^{m}} \times d f: S^{m} \times T X \rightarrow S^{m} \times f^{*} T \Sigma$ restricted to $\hat{X}$. Let each $W_{x}$ equal $\hat{X}$, with $J_{0}$ on $E \mid W_{x}$, and let $C=S^{m} \times K \cup \partial \hat{X}$, with $J_{C}$ given by $J_{0}$ near $S^{m} \times K$ and $J_{1}$ near $\partial \hat{X}$. We obtain an $\omega$-tame, $\left(\omega_{\Sigma}, f\right)$-compatible structure $J$ on $\hat{X}$ that agrees with $J_{1}$ on $\partial \hat{X}$. Since $L$ and $L^{\perp}$ are $J_{1}$-complex on $\partial \hat{X}$, we can extend $J$ over $S^{m} \times X$ by declaring $L$ and $L^{\perp}$ to be complex line bundles outside $\hat{X}$ as well. Replacing $S^{m}$ by a point in this argument gives the required $J$ on $X$ completing the proof of (b). (To smooth $J$ if desired, assume it agrees with the smooth $J_{0}$ near $K$, then perturb away from $K$ by first smoothing a $J$-complex line bundle complementary to $L$.) On the other hand, Lemma 3.2(a) (over $D^{m+1} \times X$ with $C=S^{m} \times X$ ) extends $J$ to an $\left(\omega_{\Sigma}, f\right)$-compatible structure on $D^{m+1} \times X$ that is $\omega$-tame on $\partial D^{m+1} \times X$. As in the uniqueness proof of Theorem 2.7(a) (which is the $m=0$ case), we can now construct a $J$-taming symplectic form on each $\{p\} \times X$ (Theorem 1.5), then splice by a partition of unity on $D^{m+1}$ to obtain a family of forms parametrized by $D^{m+1}$. The resulting map $D^{m+1} \rightarrow \mathcal{S}$ provides a nullhomotopy of the original map, showing $\pi_{m}(\mathcal{S})=0$ for all $m$. But $\mathcal{S}$ is an open subset of a metrizable vector space of closed forms, so it is an ANR and hence contractible [8] (cf. last paragraph of [4, Section 3]).

Proof of Lemma 3.3. The lemma is obvious when $n=1$, so we prove the statement for fixed $n>1$, inductively assuming it for $n-1$. It is well-known that if $G$ is a finite, connected graph for which each edge has two distinct 
vertices, then there is a vertex $v$ with $G-\{v\}$ connected. (This is easy to prove by induction on the number of vertices: Fix a pair of vertices connected by an edge, delete all edges between them and identify the pair, then apply the induction hypothesis to this smaller graph.) Given $A$ satisfying (a)(c), reorder the coordinates so that $G_{A}-\left\{v_{n}\right\}$ is connected. If (d) fails, then $\operatorname{rank} A<n$, but the $(n-1) \times(n-1)$ matrix obtained from $A$ by deleting the last row and column has rank $n-1$ by induction. (Hypothesis (d) holds for it since connectivity of $G_{A}$ implies $a_{n j}>0$ for some $j<n$.) Thus, we can assume $A$ satisfies (a)-(d). Note that $a_{n n}<0$ by (a), (b) and (c). Eliminate the remaining entries in the last column by row operations, adding $-a_{i n} / a_{n n}$ times the $n^{\text {th }}$ row to the $i^{\text {th }}$ row for $i<n$, then delete the last row and column and let $B=\left[b_{i j}\right]$ denote the resulting $(n-1) \times(n-1)$ matrix. Since $b_{i j}=a_{i j}-\frac{a_{i n} a_{n j}}{a_{n n}}, B$ is symmetric, so it now suffices to show $B$ satisfies (a)-(d). Condition (b) is obvious since $b_{i j} \geq a_{i j}$ for all $i, j<n$. Condition (a) follows since $G_{B}$ is obtained from $G_{A}$ by deleting $v_{n}$ (and adjacent edges) and possibly adding edges. Condition (c) follows from two applications of (c) for $A$. First, we have (for $j=n$ )

$$
\sum_{i=1}^{n-1} m_{i} a_{i n} \leq-m_{n} a_{n n}
$$

Since $a_{n n}<0$, we obtain $\sum_{i=1}^{n-1} m_{i} b_{i j}=\sum_{i=1}^{n-1} m_{i} a_{i j}-\left(\sum_{i=1}^{n-1} m_{i} a_{i n}\right) \frac{a_{n j}}{a_{n n}} \leq$ $\sum_{i=1}^{n-1} m_{i} a_{i j}+m_{n} a_{n j}=\sum_{i=1}^{n} m_{i} a_{i j} \leq 0$ for all $j<n$, verifying (c). If the final inequality here is strict for some $j<n$, then (d) follows and we are finished. Otherwise, (d) for $A$ implies that the displayed inequality (for which $j=n$ ) is strict. By (a), there is some $j<n$ for which $a_{n j} \neq 0$, so the remaining inequality above is strict for this $j$, and (d) follows. (Note that condition (a) is crucial. Otherwise, a matrix of diagonal blocks satisfying (b), (c) but not (d) would be a counterexample.)

\section{References.}

[1] S. Donaldson, Lefschetz fibrations in symplectic geometry. Doc. Math. J. DMV, Extra Volume ICMII (1998), 309-314.

[2] R. Fintushel and R. Stern, Knots, links and 4-manifolds, Invent. Math. 134 (1998), 363-400.

[3] R. Gompf, A new construction of symplectic manifolds. Ann. of Math. 142 (1995), 527-595. 
[4] R. Gompf, Toward a topological characterization of symplectic manifolds, J. Sympl. Geom. 2 (2004), 177-206.

[5] R. Gompf and A. Stipsicz, 4-manifolds and Kirby calculus. Grad. Studies in Math. 20, Amer. Math. Soc., Providence, (1999).

[6] T.-J. Li and A.-K. Liu, Uniqueness of symplectic canonical class, surface cone and symplectic cone of 4 -manifolds with $b^{+}=1$, J. Diff. Geom. 58 (2001), 331-370.

[7] C. McMullen and C. Taubes, 4-manifolds with inequivalent symplectic forms and 3-manifolds with inequivalent fibrations, Math. Res. Lett. 6 (1999), 681-696.

[8] R. Palais, Homotopy theory of infinite dimensional manifolds, Topology 5 (1966), 1-16.

[9] I. Smith, On moduli spaces of symplectic forms, Math. Res. Lett. 7 (2000), 779-788.

[10] Z. Szabó, Simply connected irreducible 4-manifolds with no symplectic structures, Invent. Math. 132 (1998), 457-466.

[11] C. Taubes, The Seiberg-Witten invariants and symplectic forms. Math. Res. Letters 1 (1994), 809-822.

[12] W. Thurston, Some simple examples of symplectic manifolds. Proc. Amer. Math. Soc. 55 (1976), 467-468.

Department of Mathematics

The University of Texas at Austin

1 University Station C1200

Austin, TX 78712-0257

E-mail address: gompf@math.utexas.edu

Received June 2, 2004. 